ITC 1/48

Journal of Information Technology and Control

Vol. 48/ No. 1/ 2019

pp. $129-145$

DOI 10.5755/j01.itc.48.1.17270
An Authentication Framework for Roaming Service in Global Mobility Networks

\title{
An Authentication Framework for Roaming Service in Global Mobility Networks
}

\section{Jangirala Srinivas}

Jindal Global Business School, O. P. Jindal Global University, Haryana 131 001, India

\section{Dheerendra Mishra}

Department of Mathematics, The LNM Institute of Information Technology, Jaipur, India; e-mail: dheerendra.mishra@lnmiit.ac.in

\section{Sourav Mukhopadhyay}

Department of Mathematics, Indian Institute of Technology, Kharagpur 721 302, India

\section{Saru Kumari}

Department of Mathematics, Ch. Charan Singh University, Meerut, India

\section{Vandana Guleria}

Department of Mathematics, Birla Institute of Technology, Mesra, Ranchi, India

Corresponding author: dheerendra.m@gmail.com

In global mobility networks (GLOMONET), to provide secure and privacy-preserving communication among authorized mobile users in roaming services is not an easy task. To achieve authorized communication, mutual authentication is performed among legal users in GLOMONET. Therefore, security as well as privacy should be addressed in designing the security protocols for GLOMONET. In recent years, most of the research work is focused on one-way authentication and does not have desirable security attributes. In this paper, we discuss the development of authentication protocol for GLOMONET. To address security and privacy issues in authorized communication, we proposed a provably secure authentication protocol for GLOMONET. To identify the resistance against known attacks, we have analyzed the scheme against all known attacks. The comparative study on the security and performance with the related results manifests that the proposed scheme addresses the security and privacy challenges and avails comparable performance.

KEYWORDS: Global mobility networks, authentication, anonymity, untraceability, security. 


\section{Introduction}

Global Mobility Networks (GLOMONET) provide the roaming services for the mobile users, which enable them to use the extended services in their home agent whenever they enter into a foreign agent zone irrespective of their locations [2]. When a mobile user $(M U)$ enters a foreign agent zone, there should be some mechanism of communication between a mobile user, home agent ( $H A)$, and foreign agent $(F A)$. A conventional frame for roaming service is shown in Figure 1. To communicate with $F A$, firstly, a session is developed with the home agent by $M U$. Then, $M U$ communicates with $F A$ with the help of $H A$. A registered $M U$ gets the services only when he/she is successfully authenticated by FA. Meanwhile, in practice, two major concerns (privacy and security) are identified in roaming services. Thus, in attaining the roaming services, the mutual authentication mechanism is designed to address the issues of authorized communication. The secure communication is being achieved using key agreement $[1,21,27]$. As the communication technologies are developing rapidly, the entire world is reciprocally more connected. Due to this privacy risks are at stake. Privacy concerns have increasingly got attention from governments, corporations, and individuals. It is desirable to control sensitive information. However, in the environment of Internet where information sharing is very easy, this problem (controlling sensitive information) does not have an easy solution. Thus, a balanced approach is required between information sharing and privacy.

For roaming services in GLOMONET, the first authentication scheme was introduced by Zhu and Ma
[36]. Since then, there were many proposals for a secure design with low computation cost $[6,10-11$, $18-19,29,31]$. It is observed that most of the existing similar schemes failed to provide the user anonymity along with untraceability.

In 2011, two new authentication schemes were proposed by Chen et al. [4-5]. However, Xie et al. [33] enlightened the security shortcomings of both the schemes. For roaming services, Mun et al. [22] gave an anonymous authentication scheme in 2012. However, Mun et al.'s scheme is shown failure by Kim and Kwak [15] due to the design flaws in their scheme.

Based on quadratic residue, Jiang et al. [13] gave an authentication scheme. But, Wen et al. [30] and He et al. [12] both pointed out that service attack, replay attack and impersonation attack are not addressed in Jiang et al.'s scheme. To overcome the security flaws, Wen et al. [30] and He et al. [12] independently presented improved schemes. Using the modular exponentiation operations, Shin et al. [26] gave an efficient authentication scheme. Unfortunately, Farash et al. [9] pointed out that both [30] and [26] failed to resist user traceability, impersonation attack and session key disclosure attack. Due to these failures, a lightweight authentication scheme has been presented by them.

Recently (2015), Zhang et al. [34] designed an authentication scheme by adopting symmetric key and rational points multiplication in elliptic curve to preserve the privacy in GLOMONET. As a part of our case study, we figured out that their scheme fails to ensure the security goals. We came across some security shortcomings

\section{Figure 1}

Conventional frame for roaming services

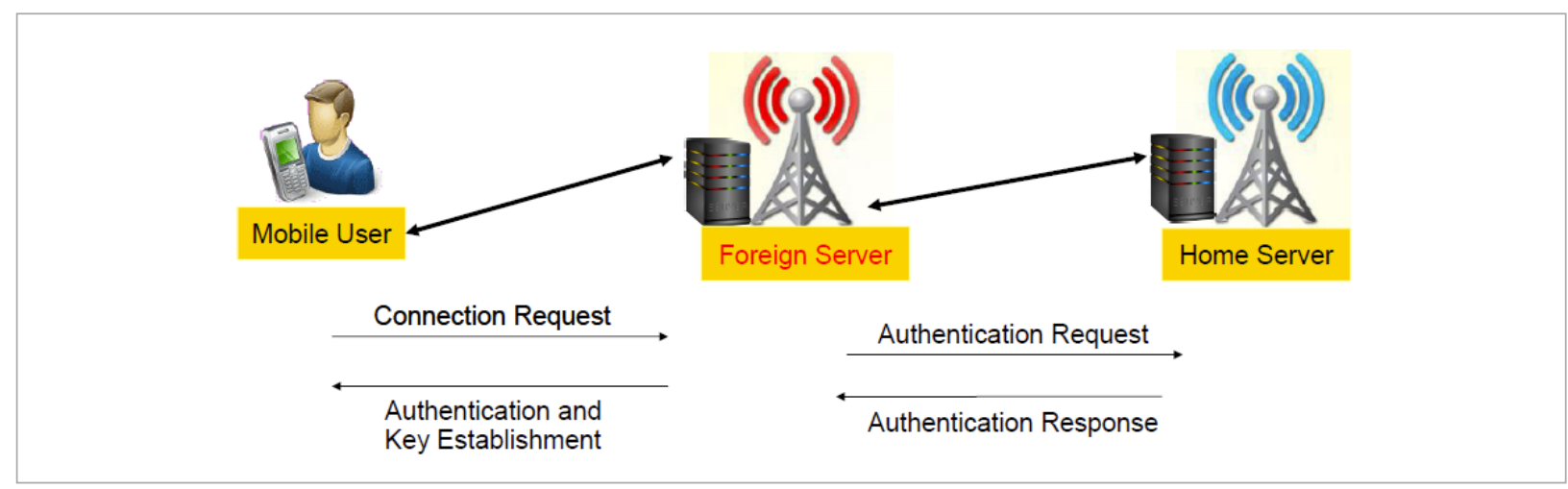


in Zhang et al.'s scheme, such as (a) password guessing attack; (b) user anonymity and traceability; (c) replay attack; and (d) user impersonation attack. In a thorough study, we observed that Zhang et al.'s scheme can be enhanced such that improved design can collectively address all the security attributes.

\subsection{Security Goals}

In providing secure roaming services, an efficient and anonymous authentication scheme should possess the following attributes:

Mobile user's privacy: Roaming authentication has issues with two types of privacy: (i) MU's real identity; and (ii) MU's roaming line. Both types should be required to provide privacy when running the roaming services.

Untraceability: No tracking of $M U$ by looking at the connections, i.e., the third party must not be able to identify $M U$ by looking at his interactions with different $F A$ s.

User's validation checking: The registration of $M U$ with $H A$ should be correctly identified by the foreign agent.

Prohibit impersonation attack: Only the legitimate mobile user and the network agent (home or foreign) should be able to authenticate.

Ensure a private session key: The parties should agree upon a fresh session key. The session key can be established among the authorized participants.

Prohibit replaying attack: Interception of the messages by attacker should not reveal any sensitive information of the participants even by replaying the previous messages.

\subsection{Our Contributions}

The contributions are as follows:

- In a thorough study of the recently proposed Zhang et al.'s [34] scheme, we concluded that their scheme suffers from several attacks such as (a) password guessing attack; (b) user anonymity and traceability; (c) replay attack; and (d) user impersonation attack.

- To overcome the shortcomings of Zhang et al.'s scheme, we present an improved scheme which inherits Zhang et al.'s scheme and successfully withstands the possible known attacks.

- Moreover, the proposed scheme is proved secure assuming the hardness of ECDH assumption.
The scheme is presented with a valid proof which preserves all the security attributes.

- Furthermore, the proposed scheme is computationally efficient in comparison to Zhang et al.'s scheme and performs better in comparison to other existing similar schemes.

\subsection{Cryptographic Preliminaries}

\subsubsection{Elliptic Curve}

An elliptic curve $E$ over a finite field $F_{P}$ consists of points satisfying the equation $y^{2}=x^{3}+a x+b \bmod p$ along with the point at infinity, where $a, b \in F_{p}$ and $4 a^{3}+27 b^{2} \bmod p \neq 0$. We omit $\bmod p$ and draw out the following assumptions [17].

\section{Assumption1:}

Ellipticcurvediscretelogarithmproblem(ECDLP): Suppose $P, Q \in G$ with $Q=\alpha P$, it is computationally hard to compute the integer $\alpha$, where $G$ is the group of rational points on the elliptic curve $E\left(F_{p}\right)$.

\section{Assumption2:}

EllipticcurveDiffie-Hellmanproblem(ECDHP):

If $\alpha P, \beta P \in G$ and $\alpha, \beta$ are positive integers, it is hard to compute $\alpha \beta P$.

\subsubsection{Biohashing}

Biometric systems are applicable for human authentication in validating the security task to enable the authorized access, however, these systems face specific security challenges such as noisy data input which causes denial of service attack. It has impact on the usability of the system by failing to identify authorized consumers [23]. To overcome these problems, BioHashing technique is introduced [28]. It has substantial functional advantages such as clean separation of the genuine, zero error rate and imposter populations [14]. It has the following functions:

- Extraction of biometric parameter represented in a vector form $\Gamma \in \mathcal{R}^{n}$, where $n$ is the feature length.

- Input token is used to generate $m$ pseudo-random vector $\left\{r_{i} \in \mathcal{R}^{M} \mid i=1,2, \cdots m\right\}$.

- The Gram-Schmidt process is employed for $\left\{r_{i} \in \mathcal{R}^{M} \mid i=1,2, \cdots m\right\} \quad$ and orthonormal pseudorandam vectors are obtained $\left\{r \perp i \in \mathcal{R}^{n} \mid i=1,2, \cdots m\right\}, n \geq m$.

_ Calculate $\{\langle\Gamma \mid r \perp i\rangle \mid i=1,2, \cdots m\}$, where $\langle. \mid$. indicates inner product. 
- Compute $m$-bit BioHash template, $b=$ $\left\{b_{i} \mid i=i=1,2, \cdots m\right\}$ using a threshold $\kappa$ obtained from

$$
b_{i}= \begin{cases}1, & \text { if }\langle\Gamma \mid r \perp i\rangle \leq \kappa \\ 0, & \text { if }\langle\Gamma \mid r \perp i\rangle>\kappa\end{cases}
$$

\subsection{Road Map of the Paper}

The details are as follows. In Section 2, we present the review of Zhang et al.'s scheme. Security weaknesses of Zhang et al.'s scheme are shown in Section 3. The proposed authentication scheme is provided in Section 4. Further, in Section 5, we present our provably secure scheme in formal model.

The informal security analysis and discussion of the proposed scheme is done in Section 6. In Section 7, the performance of our scheme with the other existing similar schemes is compared. Finally, the paper is concluded in Section 8.

\section{Review of Zhang et al.'s Scheme}

\subsection{Registration Phase}

$M U$ registers with the home agent $H A$ if he/she wishes to get services from Global Mobility Network [34]. In this phase, the communication with the participants is done through secure communication channel.

R1. $M U$ selects his/her identity $I D I D_{M U}$ and password $P W_{M U}$. After computing $V=h\left(P W_{M U} \| m\right), M U$ sends the message $\left\{I D_{M U}, V\right\}$ to home agent $H A$ where $m \in Z_{p}^{*}$ is a random number.

R2. $H A$ receives the message $\left\{I D_{M U}, V\right\}$ and a random number $n$ is generated to undergo computations using $H A$ 's master key $K, M I D=E n c_{K}\left(I D_{M U}, n\right)$, $C=V \oplus h\left(I D_{M U} \| K\right)$. Further $H A$ sends $\left\{M I D, C, I D_{H A}\right\}$ to $M U$.

R3. $M U$ stores $\left\{I D_{M U}, I D_{H A}, C, M I D, m\right\}$ into $S C$.

\subsection{Authentication and Key Establishment Phase}

The registered $M U$ opts services from $F A$. Before this, both the parties agree upon a common session key. Moreover, the communication between $F A$ and $H A$ is done through the secure channel. The details are shown as follows:
A1. MUinputshis/herlogin credentialsinto the smartcard to compute $V=h\left(P W_{M U} \| m\right)$ and $V^{\prime}=C \oplus V$. Further, $M U$ chooses a random number $a \in Z_{p}^{*}$ and computes $a P$ and $A u t h_{M U}=E n c_{V^{\prime}}\left(I D_{M U}, a P\right) . M U$ sends the message $m_{1}=\left\{I D_{H A}, M I D\right.$, Auth $\left._{M U}\right\}$ to the foreign agent $F A$.

A2. $F A$ receives the message $m_{1}$, and selects a random number $b \in Z_{p}^{*}$, computes $b P$ and $D_{F A}=E n c_{K_{F H}}\left(I D_{F A}, b P, T_{F A}\right)$, where $T_{F A}$ is $F A^{\prime} s$ chosen timestamp, and $K_{F H}$ is a pre-shared secret known to $F A$ and $H A$. The message $m_{2}=\left\{I D_{F A}, M I D\right.$, Auth $\left._{M U}, T_{F A}, D_{F A}\right\}$ is sent to $H A$.

A3. $H A$ receives the message $m_{2}$, decrypts $M I D$ and $D_{F A}$ to obtain $I D_{M U}, T_{F A}$. $H A$ verifies the correctness of $I D_{M U}$ and $T_{F A}$. If the verification doesn't hold, $H A$ rejects the process. Else, $H A$ calculates $V^{\prime}=h\left(I D_{M U} \| K\right)$ using HA's master key and decrypts Auth $_{M U}$ to fetch aP. A random number $n^{\prime}$ is generated by $H A$ to compute $M I D^{\prime}=E n c_{K}\left(I D_{M U}, n^{\prime}\right)$, $D_{H A}=E n c_{K_{F H}}\left(I D_{H A}, I D_{M U}, a P, b P\right)$ and Auth $_{H A}=$ $E n c_{V^{\prime}}\left(M I D^{\prime}, a P, b P\right)$, where $T_{H A}$ is a timestamp of $H A$. Finally, the message $m_{3}=\left\{D_{H A}, A u t h_{H A}\right\}$ is sent to $F A$.

A4. $F A$ receives the message $m_{3}$ and decrypts $D_{H A}$ and looks for the correctness of $b P$ and $T_{H A}$. If the correctness holds, FA computes Auth $_{F A}=h\left(a b P\left\|I D_{M U}\right\| I D_{F A}\right)$ $S K_{M F}=h\left(a P\|b P\| a b P\left\|I D_{M U}\right\| I D_{F A}\right)$. The message $m_{4}=\left\{\right.$ Auth $_{F A}$, Auth $\left._{H A}\right\}$ is sent to $M U$.

A5. To obtain $a P, b P$ and $M I D^{\prime}$, MU decrypts $A u t h_{H A}$. $M U$ verifies whether the decrypted $a P$ is same as that of the value in step A1. If the verification holds with the chosen secret value $a$, then $M U$ needs to verify the correctness of $A u t h_{F A}$ to compute the session key $S K_{M F}=h\left(a P\|b P\| a b P\left\|I D_{M U}\right\| I D_{F A}\right)$. The smartcard updates $M I D$ with $M I D^{\prime}$ in its memory.

\subsection{Update Session Key}

Interested readers can refer to [34].

\section{Security Pitfalls of Zhang et al.'s Scheme}

\subsection{Adversary Model}

The security of Zhang et al.'s scheme is analyzed under the following security model $[7-8,16,20]$ :

1 Over the public channel, Adversary/Attacker $(\mathcal{A})$ has the ability to eavesdrop all the communica- 
tions between the parties.

$2 \mathcal{A}$ attains the potential to delete, modify, resend or to redirect the eavesdropped transmitted messages.

3 By analyzing the method of power analysis and consumption or from the leaked information, the information from the smart card can be extracted by $\mathcal{A}$.

$4 \mathcal{A}$ can be an insider of the system.

\subsection{Disadvantages:}

1 No verification mechanism for user's login credentials to check the legitimacy of the user.

2 The communication message $m_{1}$ is transmitted to $F A$ even if illegal credentials (Wrong credentials) are being used by a user/adversary.

3 Incorrect login credentials induce huge communication and computational wastage.

\subsection{Stolen Smartcard Attack}

Suppose that the smartcard of $M U$ is lost or stolen for a deliberate amount of time and replaced. The adversary $\mathcal{A}$ can get control over the smartcard as discussed in Section 3.1. Using this captured smartcard parameters and the transmitted messages on the insecure channel, $\mathcal{A}$ can perform the following attacks:

\subsubsection{Password Guessing Attack}

1 Initially, $\mathcal{A}$ guesses the $P W_{M U}^{\mathcal{A}}$ and computes $V^{\mathcal{A}}=h\left(P W_{M U}^{\mathcal{A}} \| m\right), V^{\prime}=V^{\mathcal{A}} \oplus C$.

$2 \mathcal{A}$ decrypts $A u t h_{M U}$ and $A u t h_{H A}$ using the computed $V^{\prime}$.

$3 \mathcal{A}$ verifies the parametric value of $a P$ in both $A u t h_{M U}$ and $A u t h_{H A}$ from the above decryption. If the value is the same in both the decryption computations, $\mathcal{A}$ guesses the password of the $M U$ successfully.

4 Otherwise, $\mathcal{A}$ repeats the above steps until the password $P W_{M U}$ is guessed correctly.

Therefore, $\mathcal{A}$ will be able to guess the $M U$ s password.

\subsubsection{User Anonymity and Traceability}

According to Subsection 3.3.1, adversary guesses the password of $M U$ and computes $V^{\prime}$. Further $\mathcal{A}$ decrypts Auth $_{M U}$ using $V^{\prime}$. It is evident that the decryption of $A_{u t h}$ discloses the identity $I D_{M U}$ of the $M U$. Thus, $I D_{M U}$ allows $\mathcal{A}$ to differentiate the users in every different login sessions which may breach the privacy of the user. Therefore, we claim that privacy of the user was not taken proper care as refered in [34].

\subsection{User Impersonation Attack}

We have seen that $\mathcal{A}$ is able to compute $V^{\prime}$ (i.e., $\left.V^{\prime}=h\left(I D_{M U} \| K\right)\right)$ successfully. Using the adversary capabilities described in Section 3.1 and $V^{\prime}, \mathcal{A}$ modifies Auth $_{M U}$ using his selected random number (say $\left.\alpha \in Z_{P}^{*}\right)$. The details are as follows:

$1 \mathcal{A}$ computes $a P$ and $A u t h_{\mathcal{A}}=E n c_{V^{\prime}}\left(I D_{M U}, \alpha P\right)$ and transmits the message $m_{1}=\left\{I D_{H A}, M I D, A u t h_{\mathcal{A}}\right\}$ to FA.

$2 F A$ receives the message $m_{1}$ and selects a random number $b \in Z_{p}^{*}$ computes $b P$ and $D_{F A}=$ $E_{n c_{K_{F H}}}\left(I D_{F A}, b P, T_{F A}\right)$. The message $m_{2}=$ $\left\{I D_{F A}, M I D, A u t h_{\mathcal{A}}, T_{F A}, D_{F A}\right\}$ is sent to $H A$.

3 On receiving $m_{2}, H A$ decrypts $M I D$ and $D_{F A}$ to obtain $I D_{M U}$ and $T_{F A}$. $H A$ verifies the correctness of $I D_{M U}$ and $T_{F A}$. If verification fails, $H A$ rejects the process. Else, $\mathcal{A}$ makes use of $H A^{\prime}$ 's master key $K$, computes $V^{\prime}=h\left(I D_{M U} \| K\right)$ and decrypts $A u t h_{\mathcal{A}}$ to fetch $\alpha P, T_{1}$. Then a random number $n^{\prime}$ is chosen from $Z_{p}^{*}$ to compute $D_{H A}=E n c_{K_{F H}}\left(I D_{H A}, I D_{M U}, \alpha P, b P, T_{1}, T_{H A}\right) \quad$ and Auth $_{H A}=E_{n C_{V^{\prime}}}\left(M I D^{\prime}, \alpha P, T_{1}, b P\right)$. Finally, the message $m_{3}=\left\{D_{H A}\right.$, Auth $\left.{ }_{H A}\right\}$ is sent to $F A$.

$4 F A$ receives the message $m_{3}$ and decrypts $D_{H A}$ and looks for the correctness of $b P$ and $T_{H A}$. If the correctness holds, FA computes $A u t h_{F A}=h\left(\alpha b P\left\|I D_{M U}\right\| I D_{F A}\right), \quad S K_{M F}=$ $h\left(\alpha P\|b P\| \alpha b P\left\|I D_{M U}\right\| I D_{F A}\right)$. The message $m_{4}=\left\{A u t h_{F A}, A u t h_{H A}\right\}$ is sent to $M U$.

$5 \mathcal{A}$ captures the message $m_{4}$.

The above procedure indicates that the adversary can successfully impersonate $M U$ by making both $F A$ and $H A$ believe that they are communicating with $M U$.

\subsection{Replay Attack}

The adversary $\mathcal{A}$ can capture the previously communicated messages as described in Section 3.1. $\mathcal{A}$ uses the communicated messages and replays the same message (say $m_{1}$ ) to $F A$ and the same is transmitted to $H A$ via $F A$ as there is no fresh verification of the messages sent by $M U$ at $H A$. Therefore, Zhang et al.'s scheme does not prevent $\mathcal{A}$ from sending the replay messages.

\subsection{Absence of Unauthorized Login Detection}

A user may sometime fail to correctly map different password to his/ her different accounts. Thus, login credential verification should be supported at initial 
stage. However, Zhang et al.'s scheme lacks user credential verification mechanism in login phase. Lack of efficient login phase makes the scheme inefficient.

\section{The Proposed Scheme}

In proposed scheme, $K_{H} \in Z_{p}^{*}$ is the secret key of $H A$ and $K_{F} \in Z_{p}^{*}$ is the secret key of FA. FA and $H A$ also share a common secret key $K_{F H}$. Furthermore, $F A$ and $H A$ also compute their public keys $K_{H} P$ and $K_{F} P$, respectively.

\subsection{Registration Phase}

This phase utilizes the secure channel to communicate with the participants. $M U$ registers with $H A$, if he/she wishes to get services from Global Mobility Network.

$1 M U$ selects his/her identity $I D_{M U}$, password $P W_{M U}$, computes $Y_{i}=h\left(P W_{M U} \| m\right)$ and transmits the message $\left\{I D_{M U}, Y_{i}\right\}$ to home agent, where $m \in Z_{p}^{*}$ is a random number.

$2 H A$ receives the message $\left\{I D_{M U}, Y_{i}\right\}$ and

\section{Table 1}

Login, authentication and key agreement phase of our scheme

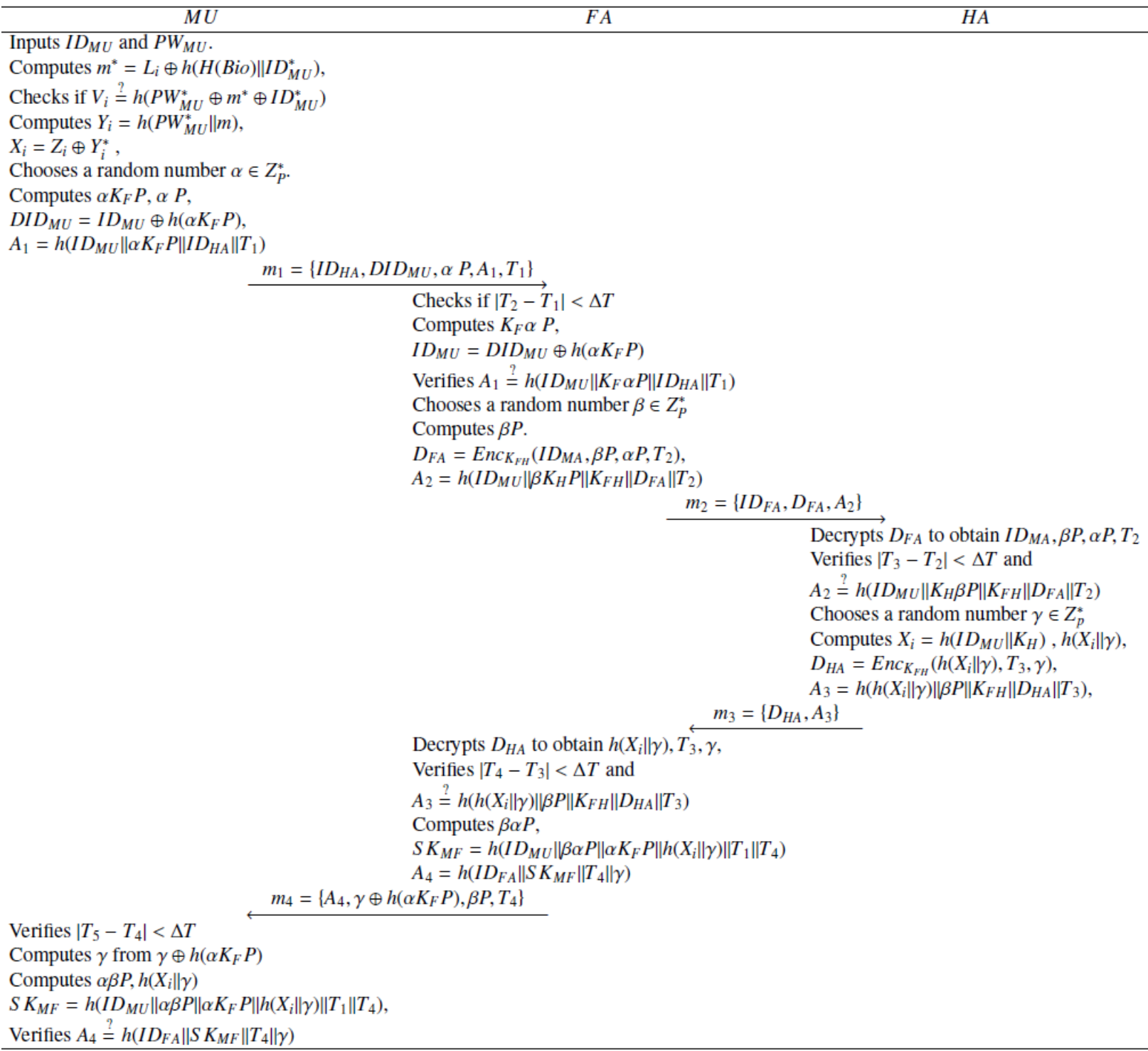


generates a random number $n$. $H A$ performs computations using its master key $K$. HA computes $X_{i}=h\left(I D_{M U} \| K_{H}\right), Z_{i}=Y_{i} \oplus X_{i}$. Further, $H A$ stores $\left\{Z_{i}, I D_{H A}\right\}$ into the smartcard $S C$ and sends to $M U$.

$3 M U$ imprints his/her biometric Bio and computes $V_{i}=h\left(P W_{M U} \oplus m \oplus I D_{M U}\right), \quad L_{i}=h(H($ Bio $) \|$ $\left.I D_{M U}\right) \oplus m . M U$ stores $L_{i}, V_{i}$ in $S C$.

\subsection{Login, Authentication and Key Establishment Phase}

In this phase, the communication between $F A$ and $H A$ is done through the secure channel. The registered $M U$ opts services from $F A$. Before this, both the parties agree upon a common session key which is described as follows:

1 MUinputs his/her login credentials into the smartcard to compute $m^{*}=L_{i} \oplus h\left(H(B i o) \| I D_{M U}^{*}\right) M U$ verifies $V_{i} \stackrel{?}{=} h\left(P W_{M U}^{*} \oplus m^{*} \oplus I D_{M U}^{*}\right)$ If verification holds, $M U$ computes $Y_{i}=h\left(P W_{M U}^{*} \| m\right)$, and $X_{i}=Z_{i} \oplus Y_{i}^{*}$. $M U$ randomly selects $\alpha \in Z_{p}^{*}$ and timestamp $T_{1}$ and computes $\alpha K_{F} P, a P, D I D_{M U}=I D_{M U} \oplus h\left(\alpha K_{F} P\right)$, $A_{1}=h\left(I D_{M U}\left\|\alpha K_{F} P\right\| I D_{H A} \| T_{1}\right) . M U$ sends the message $m_{1}=\left\{I D_{H A}, D I D_{M U}, \alpha P, A_{1}, T_{1}\right\}$ to $F A$.

2 Once $F A$ receives the message $m_{1}$ at time $T 2$, $F A$ checks $\left|T_{2}-T_{1}\right|<\Delta T$. If the verification fails, $F A$ rejects the query. Otherwise, $F A$ computes $K_{F} \alpha P$, and $I D_{M U}=D I D_{M U} \oplus h\left(\alpha K_{F} P\right)$. FA verifies $A_{1} \stackrel{?}{=} h\left(I D_{M U}\left\|K_{F} \alpha P\right\| I D_{H A} \| T_{1}\right)$. If verification holds, $F A$ selects $\beta \in Z_{p}^{*}$ randomly and computes $\beta P$ and $D_{F A}=E n c_{K_{F H}}\left(I D_{M A}, \beta P, \alpha P, T_{2}\right)$, where $K_{F H}$ is a pre-shared secret held between $F A$ and HA. FA sends the message $m_{2}=\left\{I D_{F A}, D_{F A}, A_{2}\right\}$ to $H A$, where $A_{2}=h\left(I D_{M U}\left\|\beta K_{H} P\right\| K_{F H}\left\|D_{F A}\right\| T_{2}\right)$.

3 Upon receiving the message $m_{2}$ from $F A$ at time $T_{3}, H A$ decrypts $D_{F A}$ to obtain $I D_{M A}, \beta P, \alpha P, T_{2} . \quad H A$ verifies $\quad\left|T_{3}-T_{2}\right|<\Delta T$ and $\quad A_{2}=h\left(I D_{M U}\left\|K_{H} \beta P\right\| K_{F H}\left\|D_{F A}\right\| T_{2}\right)$. If the verification does not hold, $H A$ rejects the process. Otherwise, $H A$ selects $\gamma \in Z_{p}^{*}$ and computes $X_{i}=h\left(I D_{M U} \| K_{H}\right)$ and $h\left(X_{i} \| \gamma\right)$. $H A$ sends the message $m_{3}=\left\{D_{H A}, A_{3}\right\}$ to $F A$, where $D_{H A}=E n c_{K_{F H}}\left(h\left(X_{i} \| \gamma\right), T_{3}, \gamma\right) \quad$ and $A_{3}=h\left(h\left(X_{i} \| \gamma\right)\|\beta P\| K_{F H}\left\|D_{H A}\right\| T_{3}\right)$.

$4 F A$ receives the message $m_{3}$ at time $T_{4}, F A$ decrypts $D_{H A}$ to obtain $h\left(X_{i} \| \gamma\right), T_{3}, \gamma$.FA looks for the correctness of $A_{3} \stackrel{?}{=} h\left(h\left(X_{i} \| \gamma\right)\|\beta P\| K_{F H}\left\|D_{H A}\right\| T_{3}\right)$ and $\left|T_{4}-T_{3}\right|<\Delta T$. The process termi- nates $F A$, if the verification fails. Otherwise, $F A$ computes $\beta \alpha P$, the session key $S K_{M F}=h\left(I D_{M U}\|\beta \alpha P\| \alpha K_{F} P\left\|h\left(X_{i} \| \gamma\right)\right\| T_{1} \| T_{4}\right)$ and $A_{4}=h\left(I D_{F A}\left\|S K_{M F}\right\| T_{4} \| \gamma\right)$. FA sends the message $m_{4}=\left\{A_{4}, \gamma \oplus h\left(\alpha K_{F} P\right), \beta P, T_{4}\right\}$ to $M U$.

5 On receiving the message $m_{4}$ at time $T_{5}, M U$ verifies $\left|T_{5}-T_{4}\right|<\Delta T$. If verification holds, $M U$ computes $\gamma$ from $\gamma \oplus h\left(\alpha K_{F} P\right)$. $M U$ computes $\alpha \beta P, h\left(X_{i} \| \gamma\right) \quad$ and $\quad S K_{M F}=h\left(I D_{M U}\|\alpha \beta P\|\right.$ $\left.\alpha K_{F} P\left\|h\left(X_{i} \| \gamma\right)\right\| T_{1} \| T_{4}\right)$. The mutual authentication succeeds if the verification $A_{4} \stackrel{\text { ? }}{=}$ $h\left(I D_{F A}\left\|S K_{M F}\right\| T_{4} \| \gamma\right)$ holds.

The illustration of this phase is shown in Table 3.

\subsection{Update Session Key}

Assume that $M U$ stays in the $F A$ network zone for consecutive sessions. In this scenario, this would be inefficient for $M U$ to perform re-authentication procedure with $F A$ and by involving $H A$ to attain a session key. So, in this case, without communicating with $H A$, both $M U$ and $F A$ undergo one-time authentication where they can update the session key using the earlier established session key.

U1: Suppose that a session key $S K_{M F_{i-1}}$ between $M U$ and $F A$ was already established. If $M U$ wants to establish another session with $F A, M U$ generates $\alpha_{i} \in Z_{p}^{*}$ and computes $\alpha_{i} P$. Further, $\alpha_{i} P$ is sent to $F A$.

U2: Upon receiving $\alpha_{i} P, F A$ selects $\beta_{i}$ and computes $\alpha_{i} \beta_{i} P$ and $S K_{M F_{i}}=h\left(\alpha_{i} \beta_{i} P\right)$. FA computes TempSK $_{M F_{i}}=f_{S K_{M F_{i}}}\left(\alpha_{i-1} \beta_{i-1} P \| \alpha_{i} \beta_{i} P\right)$ and sends $\beta_{i} P$ and $T e m p S K_{M F_{i}}$ to $M U$.

U3: Upon receiving the parameters, $M U$ computes $S K_{M F_{i}}^{\prime}=h\left(\alpha_{i} \beta_{i} P\right)$, and verifies the correctness of TempSK $K_{M F_{i}}^{\prime} \stackrel{?}{=} f_{S K_{M F_{i}}^{\prime}}\left(\alpha_{i-1} \beta_{i-1} P \| \alpha_{i} \beta_{i} P\right)$. If this verification holds, both the parties update the session key as $S K_{M F_{i}}=h\left(\alpha_{i} \beta_{i} P\right)$.

\section{Formal Security Analysis of Our Scheme}

We first present the security model and algorithm assumptions that are used in proving our scheme. We present the formal security analysis by the method of provable security $[9,32]$. 


\subsection{Security Model}

The adaptability of provable security is to evaluate the invincibility of our scheme against the wellknown attacks.

The participants are mobile user $M U \in \mathcal{M U}$, home agent $H A \in \mathcal{H} \mathcal{A}$ and foreign agent $F A \in \mathcal{F} \mathcal{A}$.

\section{Adversary Capabilities}

Let $\mathcal{A}$ controls the simulator and queries oracles to destroy the privacy of authentication or the session keys. The dictionary $\mathcal{D}$ size is a fixed constant which does not change upon the security parameter $\mathcal{K}$ which $\mathcal{A}$ tries to destroy in PPT. The security parameter $\mathcal{K}$ is the session key bit-length. On the following queries, $\mathcal{A}$ performs simulation in the oracles:

$\operatorname{Execute}\left(\Pi_{M U}^{i}, \Pi_{H A}^{k}, \Pi_{F A}^{j}\right)$ : It denotes that the adversary queries an execution of the protocol between the instances $\Pi_{M U}^{i}, \Pi_{H A}^{k}, \Pi_{F A}^{j}$ by eavesdropping and gets the access. This query model is for the passive attacks against the protocol.

Encryption/decryption $\left(\Pi_{M U}^{i}, m, t e x t\right)$ : By applying encryption query, an input message $m$ is encrypted to a ciphertext text as output. Furthermore, by applying decryption query, the cipher-text is decrypted and results in an output message $m$.

$\operatorname{Send}\left(\Pi_{E}^{i}, m\right)$ : The active attacks in the channel are carried out by the transmitted messages between the instances $\Pi_{M U}^{i}$ and $\Pi_{F A}^{j}$ which are prone to dictionary attacks, man-in-the-middle attacks, impersonation attacks, and unknown key-share attacks. $\Pi_{E}^{i}$ sends a message $m$ to the requested partner. If the message $m$ is valid, the query is accepted by the simulator. Otherwise, the session is rejected. An interaction with $\operatorname{Send}\left(\Pi_{M U}^{i}\right.$, start : $\left.\langle H A, F A\rangle\right)$ indicates that $\Pi_{M U}^{i}$ initiates a session with instances of $H A$ and $F A$.

$\operatorname{Reveal}\left(\Pi_{E}^{i}\right)$ : This query model is for known-key attacks. It outputs a terminator $\perp$, if the oracle has not been accepted. Otherwise, it outputs a session key $S K_{E}^{i}$. CorruptSC(MU)/CorruptLL $(\mathcal{M U})$ : The lost smartcard problem and the threat of smartcard breach are handled by this query model. The attacker $\mathcal{A}$ imposes offline password guessing by eavesdropping on messagees.

$\operatorname{Corrupt} L L\left(\Pi_{E}^{i}\right)$ : This query is in correspondence to strong forward security. The attacker $\mathcal{A}$ obtains all the information of $\prod_{E}^{i}$. We list the possible queries for $\mathcal{A}$ as follows:
$\operatorname{Corrupt} L L\left(\Pi_{M U}^{i}\right): \mathcal{A}$ gets all information from - the smartcard with $P W$.

Corrupt $L\left(\Pi_{H A}^{t}\right)$ : This query model is for the - privileged insider attacks.

CorruptVFR $\left(\Pi_{H A}^{t}\right)$ : In this query model, the - passwords which are stored by HA can be prone to stolen verifier attacks.

Corrupt $L L\left(\Pi_{F A}^{j}\right)$ : This query model for the long-

- lived secrets of the foreign agent FA. This can be done by modeling agent node capture attacks.

$\operatorname{TestAKE}\left(\Pi_{E}^{i}\right)$ : This query model gives the session key. A target session is chosen by $\mathcal{A}$ to challenge after multiple queries. If no session key is found for instance $S K_{E}^{i}$, it outputs $\perp$. Otherwise, a coin namely, $b$ is flipped. If $b=1$, the session key for instance, $S K_{E}^{i}$ is returned. Otherwise, it outputs a random string of the same size. For $M F-A K E$ - fresh instance, it can be queried once. $M F-$ fresh is introduced below.

We specify few definitions to illustrate our proof as shown below in Figures 1 and 2. We have given the simulation of the queries in the interest of readers:

1 Partnering: $M U$ and $F A$ creates the session key. We call $M U$ and $F A$ as partners if and only if $\quad \operatorname{sid}_{M U}=s i d_{F A}, \quad p i d_{M U}=F A, \quad p i d_{F A}=M U$, and $S K_{M U}=S K_{F A}$ are accepted by them.

2 MF-AKE-fresh: (This shows the freshness of strong forward security) This notion is defined only for $M U$ and $F A$. We say that $\Pi_{E}^{i}$ is $M F-A K E$ fresh if the following queries do not occur:

- AReveal $\left(\Pi_{E}^{i}\right)$ appears.

- AReveal $\left(\right.$ pid $\left._{\Pi_{E}^{i}}\right)$ appears.

- Before Test happens, $\operatorname{Corrupt}\left(\Pi_{E}^{i}\right)$ or Corrupt $\left(\right.$ pid $_{\Pi_{E}^{i}}$ ) has been asked.

3 Security: The adversary $\mathcal{A}$ 's advantage against our scheme $\Pi$ is the probability that $\mathcal{A}$ correctly guesses the bit $b$ generated in $\operatorname{Test}\left(\Pi_{E}^{i}\right)$ query with $M F-A K E-$ fresh $\Pi_{E}^{i}$ is accepted. The advantage of $\mathcal{A}$ is

$A d v_{\Pi}^{M F-A K E}(\mathcal{A})=2 \operatorname{Pr}\left[b=b^{\prime}\right]-1$.

Our scheme is $M F-A K E$ secure if $A d v_{\Pi}^{M F-A K E}(\mathcal{A})$ is negligibly greater than $O\left(q_{\text {send }}\right) /|\mathcal{D}|$, depending on security parameters $l_{h}, l_{r}$ and $l_{n}$. Here $q_{\text {send }}$ is the query time of $\operatorname{Send}\left(\Pi_{E}^{i}, m\right), l_{h}, l_{r}$ and $l_{n}$ are the length of hash results, length of random numbers and length of parameter $n$, repectively. 


\section{Figure 2}

Simulation of queries

On querying a hash function $\mathrm{h}(\mathrm{m})$, if there exists a record $(\mathrm{m}, \mathrm{n})$ in $L_{h}$, an output $n$ is returned

else, the simulator chooses a random bit string $n \in\{0,1\}^{l}$, and responses as $n$ and puts $(m, n)$ into $L_{h}$

For initiate a Send $(M U, I N I T)$ query, the following steps were done by the simulator:

Computes $m^{*}=L_{i} \oplus h\left(I D_{M U}^{*} \| P W_{M U}^{*}\right), V^{*}=h\left(P W_{M U}^{*}\left\|I D_{M U}^{*}\right\| m^{*}\right)$ and verifies $A \stackrel{?}{=} h\left(V^{*} \| I D_{M U}^{*}\right)$, If this verification is false, reverts the query.

Computes $S_{H M}=V \oplus C$, selects $\alpha \in Z_{p}^{*}$ random number and $T_{1}$

Computes $\alpha P$ and $A u t h_{M U}=\operatorname{Enc}_{S_{H M}}\left(I D_{M U}, \alpha P, T_{1}\right)$.

Returns $m_{1}=\left\{I D_{H A}, M I D, A u t h_{M U}, T_{1}\right\}$ as the answer.

For a $\operatorname{Send}\left(M U, F A^{j}, m_{1}\right)$ query, the following steps were done by the simulator:

Checks $\left|T_{F A}-T_{1}\right|<\Delta T$, If the verification fails, rejects the query.

Selects $\beta \in Z_{p}^{*}$ random number,

Computes $\beta P$ and $D_{F A}=E n c_{K_{P H}}\left(I D_{F A}, \beta P, T_{F A}\right)$,

Then answers the query with $m_{2}=\left\{I D_{F A}, M I D, A u t h_{M U}, D_{F A}\right\}$

For a $\operatorname{Send}\left(F A^{j}, H A^{t}, m_{2}\right)$ query, the following steps were done by the simulator.

Decrypt $M I D$ and $D_{F A}$ to obtain $I D_{M U}$ and $T_{F A}$. and verifies the correctness of $I D_{M U}, T_{F A}$. Computes $S_{M H}=h\left(I D_{M U} \| K\right)$,

and decrypts $A$ uth $h_{M U}$ to extract $\alpha P, T_{1}$. Selects a random number $n^{\prime} \in Z_{p}^{*}$ and computes $M I D^{\prime}=E n c_{K}\left(I D_{M U}, n^{\prime} \oplus n\right)$

$D_{H A}=\operatorname{Enc}_{K_{P H}}\left(I D_{H A}, I D_{M U}, \alpha P, \beta P, T_{1}, I D_{F A}, T_{H A}\right)$ and $A u t h_{H A}=\operatorname{Enc}_{M H}\left(M I D^{\prime}, I D_{F A}, \alpha P, T_{1}, \beta P, T_{H A}\right)$,

Then answers the query with $m_{3}=\left\{D_{H A}, A u t h_{H A}\right\}$.

For a $\operatorname{Send}\left(H A^{t}, F A^{j}, m_{3}\right)$ query, the following steps were done by the simulator.

Decrypts $D_{H A}$ and verifies the correctness of $\beta P$ and $T_{H A}$.

Computes Auth $h_{F A}=h\left(T_{H A}\|\alpha \beta P\| I D_{M U} \| I D_{F A}\right), S K_{M F}=h\left(I D_{M U}\|\alpha P\| \beta P\|\alpha \beta P\| I D_{F A}\right)$.

Then answers the query with $m_{4}=\left\{A u t h_{F A}, A u t h_{H A}\right\}$.

For a $\operatorname{Send}\left(F A^{j}, M U, m_{4}\right)$ query, the following steps were done by the simulator.

Decrypts $A$ uth $h_{H A}$ to extract $\alpha P, \beta P, I D_{F A}, T_{H A}$ and $M I D^{\prime}$. and verifies the correctness of $\alpha P$.

Computes $A u t h_{F A} \stackrel{?}{=} h\left(T_{H A}\|\alpha \beta P\| I D_{M U} \| I D_{F A}\right)$. If the verification holds

computes the session key $S K_{M F}=h\left(I D_{M U}\|\alpha P\| \beta P\|\alpha \beta P\| I D_{F A}\right)$

To perform the Execute $\left(M U, F A^{j}, H A^{t}\right)$ query, the successive computation of all Send queries are done to return the messages $\left(m_{1}, m_{2}, m_{3}, m_{4}\right)$.

To perform the Reveal $\left(I^{k}\right)$ query, if the instance $I^{k}$ has been accepted and formed a session key, returns $s k_{M U}$ or $s k_{F A}$ otherwise a $\perp$ is the answer.

To perform the Corrupt(SC) query, the $U$ 's smartcard stored information is returned.

To perform the Corrupt $\left(I^{k}\right)$ query, all the stored information of $I^{k}$ is returned.

To perform the $\operatorname{Test}\left(I^{k}\right)$ query, if the verification $I^{k}$ is not equal to $M F-f r e s h$, returns $\perp$. else, a coin $b$ is tossed

The session key is returned, if the outcome is $b=1$.

A random string of length $l$ will be returned, if the outcome is $b=0$.

4 Elliptic Curve Diffie-Hellman (ECDH) assumption: Let $P$ be a generator of $G$ and, $m P$ and $n P$ be two elements of $G$, where $m, n \in Z_{q}^{*}$ and $G$ is an additive group of prime order $q$. If $\mathcal{A}$ is successful in computing $m n P$ from $(m P, n P)$, we denote it as $A d v_{\mathcal{A}}^{E C D H}(t)$ and which can also be considered as the maximal success probability among the adversary which runs within time $t$. The ECDH assumption holds if $A d v_{A}^{E C D H}(t)$ is negligible.

\subsection{MF-AKESecurity}

Theorem 1. Consider an elliptic curve with group $G$ and let $\left|D_{P W}\right|$ be the dictionary size of the password. Let our proposed scheme be $\pi$. The advantage of an adversary is considered if $\mathcal{A}$ can compute the following within a specific time bound $t$ by taking less than send-queries time $\left(q_{\text {send }}\right)$, execute-queries time $\left(q_{\text {exec }}\right)$, hash-queries time ( $\left.q_{\text {hash }}\right)$, and encryption/decryption-queries time $\left(q_{E / D}\right)$ :

$$
\begin{aligned}
\operatorname{Adv}_{\pi}^{\mathbf{M F}-\mathbf{A K E}}(\mathcal{A}) & \leq \frac{2 q_{\text {send }}}{\left|D_{P W}\right|}+\frac{\left(q_{E / D}^{2}+q_{\text {send }}+q_{\text {exec }}\right)^{2}}{2(q-1)} \\
& +A d v_{\mathcal{A}}^{E C D H}\left(t+\left(1+q_{\text {send }}+q_{\text {exec }}\right) \cdot \tau_{G}\right)(1), \\
& +\frac{q_{\text {hash }}^{2}}{2^{l}}+\frac{2\left(q_{\text {send }}(\text { Bio })+q_{\text {send }}+q_{\text {exec }}\right)}{2^{l}}
\end{aligned}
$$

where, $\tau_{G}$ denotes the time to compute the point mul- 
tiplication in $G$ and $l$ is a security parameter string of $\{0,1\}^{l}$.

Proof: The communication among $\pi_{i, j}^{s}$ and $\pi_{j, i}^{t}$ is done by considering the definitions[1-3] which are intended to be conveyed faithfully, i.e., both $\pi_{i, j}^{s}$ and $\pi_{j, i}^{t}$ will receive the fairly established message by which both the oracles can agree upon a session key. Since, $\alpha, \beta \in Z_{p}^{*}$, the session key $S K_{M F}=h\left(I D_{M V}\|\alpha \beta P\| \alpha K_{F} P\left\|h\left(X_{i} \| \gamma\right)\right\| T_{1} \| T_{4}\right) \quad$ is considered to be random for every established session which is from the key space.

Now, to prove that our scheme meets the second and third conditions. A consecutive sequence of games $G_{i}(0 \leq i \leq 5)$ is played which starts at $G_{0}$ and ends at $G_{5}$. For each game, if $\mathcal{A}$ correctly guesses the bit $c$ by posing the Test query, then it is considered as Succ $_{i}$. Thus, the Test query is done on

$\operatorname{Diff}_{i}=\left|\operatorname{Pr}\left[\operatorname{Succ}_{i}\right]-\operatorname{Pr}\left[\operatorname{Succ}_{i-1}\right]\right|, \forall(1 \leq i \leq 5)$,

which is the difference of probability of success between $G_{i}$ and $G_{i-1}$.

Once $\mathcal{A}$ finishes the last game $G_{5}$, the $M F-A K E$ Security game can only be won with probability $1 / 2$. The detail description of the games are as follows:

Figure 3

Simulation of Send queries
Game $G_{0}$ : In this game, $\mathcal{A}$ is facilitated with several oracles such as $E / D$ oracle, hash oracle. Even the participants' instances $\pi_{i, j}^{s}$ are also available to $\mathcal{A}$. Thus, by definition of $A d v_{\pi}^{M F-A K E}$ in Section 3.1:

$A d v_{\pi}^{M F-A K E}(\mathcal{A})=2 . \operatorname{Pr}\left[\operatorname{Succ}_{0}\right]-1$.

Our goal is to substantiate that $A d v_{\pi}^{M F-A K E}(\mathcal{A})$ is negligible.

Thus, we have

$\operatorname{Adv} v_{\pi}^{M F-A K E}(\mathcal{A})=2 \cdot \operatorname{Pr}\left[\operatorname{Succ}_{5}\right]-1+2 \cdot \sum_{i=1}^{5} \operatorname{Diff}_{i}$.

Game $G_{1}$ : In this game, the $E / D$ oracle and hash oracle are simulated by maintaining a hash list and an encryption/decryption list as List $_{h}$, List $_{E / D}$. List $t_{h}$ is of the form $\langle x, h(x)\rangle \forall x$ where $x$ is the input value and $h(x) \in\{0,1\}^{l}$ is an output random value. In addition, considering all the queries such as (Send, Execute, Reveal, and Test), all the instances are simulated as in the real protocol. The result of the simulation is indistinguishable from the real attack unless the following events take place:

Event 1: The permutation property of encryption/ decryption fails which means there will be some collisions on the encryption/decryption.

Event 2: The hash function is prone to collisions.

To perform the Execute $\left(M U^{i}, F A^{j}, G^{k}\right)$ query, the successive computation of all Send queries are done to return the messages $\left(m_{1}, m_{2}, m_{3}, m_{4}\right)$.

To perform the Reveal $\left(I^{k}\right)$ query, if the instance $I^{k}$ has been accepted and formed a session key, returns $s k_{M U}$ or $s k_{F A}$ Otherwise $\mathrm{a} \perp$ is the answer.

To perform the Corrupt $(S C)$ query, the $U$ 's smartcard stored information is returned. To perform the Corrupt $\left(I^{k}\right)$ query, all the stored information of $I^{k}$ is returned. Since $\mathcal{A}$ knows some information at first, the concrete results are as follows:

(a) $I=M U: \mathcal{A}$ can retrieve all the information of user's smartcard with password $P W$.

(b) $I=H A: \mathcal{A}$ can get the secret key $\mathrm{K}$.

(c) $I=F A: \mathcal{A}$ can obtain P.

To perform the $T$ est $\left(I^{k}\right)$ query, if the verification $I^{k}$ is not equal to $M F-A K E-$ fresh, returns $\perp$.

Here $I$ is $M U$ or $F A$, like Corrupt $\left(I^{k}\right)$. Else, a coin $b$ is tossed by the simulator.

The session key is returned, if the outcome is $b=1$.

A random string of length $l$ will be returned, if the outcome is $b=0$. 
Thus, the probability of the two events is restricted to $\frac{q_{h}^{2}}{2^{l+1}}+\frac{q_{E / D}}{2(q-1)}$. i.e.,

$$
\operatorname{Diff}_{1}=\mid \operatorname{Pr}\left[\text { Succ }_{1}\right]-\operatorname{Pr}\left[\text { Succ }_{0}\right] \mid
$$$$
\leq \frac{q_{h}^{2}}{2^{l+1}}+\frac{q_{E / D}}{2(q-1)} \text {. }
$$

Game $G_{2}$ : This game stops when a collision occurs on the transcripts $\left(I D_{H A}, D I D_{M U},{ }^{*}, A_{1}, T_{1}\right),\left(I D_{M U}, *\right)$, $\left(I D_{M U},{ }^{*}, I D_{H A}, T_{1}\right)$. Otherwise, $\mathcal{A}$ undergoes the simulations where all the oracles in game $G_{1}$ function. Suppose the collisions occur this imply (this case is similar to replay attack) that $\mathcal{A}$ can easily win the game. According to the birthday paradox, the probability of this event is restricted to:

$$
\begin{aligned}
& \operatorname{Diff}_{2}=\mid \operatorname{Pr}\left[\operatorname{Succ}_{2}\right]-\operatorname{Pr}\left[\text { Succ }_{1}\right] \mid \\
& \leq \frac{\left(q_{\text {send }}+q_{\text {exec }}\right)^{2}}{2(q-1)} .
\end{aligned}
$$

Game $G_{3}$ : This game stops if $\mathcal{A}$ is successful in guessing the correct authenticate values $A_{1}, A_{2}$ without checking the corresponding hash oracles with the queries. Otherwise, $\mathcal{A}$ performs the simulations on all the oracles in game $G_{2}$. Thus $G_{3}$ and $G_{2}$ are indistinguishable unless the oracle rejects a valid authentication value. The probability of this event is restricted to:

$$
\begin{aligned}
& \operatorname{Diff}_{3}=\left|\operatorname{Pr}\left[\operatorname{Succ}_{3}\right]-\operatorname{Pr}\left[\operatorname{Succ}_{2}\right]\right| \\
& \leq \frac{q_{\text {send }}+q_{\text {exec }}}{2^{l}} .
\end{aligned}
$$

Game $G_{4}$ : This game stops if $\mathcal{A}$ is successful in guessing the password and biometric key of $M U$. Then, using the guessed password and biometric key, $\mathcal{A}$ can get the secret value $V_{i}$ and further choose $\alpha P$ as a component of the Diffie-Hellman tuple. As a result, $\mathcal{A}$ can correctly distinguish the random value and the session key. Otherwise, $\mathcal{A}$ simulates all the oracles in game $G_{3}$. To show the effectiveness of the attack, $\mathcal{A}$ should continuously attempt to guess the password of $M U$. So the probability of this event is restricted to $\frac{q_{\text {send }}}{\left|D_{P W}\right|}+\frac{q_{\text {send }(B i o)}}{2^{l}}$, i.e.,

$$
\begin{aligned}
& \operatorname{Diff}_{4}=\mid \operatorname{Pr}\left[\text { Succ }_{4}\right]-\operatorname{Pr}\left[\text { Succ }_{3}\right] \mid \\
& \leq \frac{q_{\text {send }}}{\left|D_{P W}\right|}+\frac{q_{\text {send }(B i o)}}{2^{l}}
\end{aligned}
$$

Game $G_{5}$ : This game is the same as Game $G_{4}$ except that the executions are simulated using the random self-reducibility of the Elliptic Curve Diffie-Hellman $(\mathrm{ECDH})$ problem which means one ECDH instance $(\alpha P, \beta P)$ is given to compute $\alpha \beta P$ with unknown $\alpha$ and $\beta$. Firstly, a test session is chosen. When the adversary $\mathcal{A}$ chooses the test session, we choose and win the authenticated key exchange game simulated by us. We can solve the ECDH problem by using $\mathcal{A}$ as the subroutine. More precisely, we choose $\pi_{M U, F A}^{s}$ as the test session and $\pi_{F A, M U}^{s}$ as the matching session of it. For example, $\pi_{M U, F A}^{s}$ (send, receive $)=\left\{\left(I D_{H A}, D I D_{M U}, *, A_{1}, T_{1}\right)\right\}$, $\pi_{F A, M U}^{s}($ send, receive $)=\left\{\left(I D_{H A}, D I D_{M U}, *, A_{1}, T_{1}\right),\left(I D_{M U},{ }^{*}\right)\right.$, $\left.\left(I D_{M U}, *, I D_{H A}, T_{1}\right)\right\}$. When $\mathcal{A}$ asks the Execute query or Send query to $\pi_{M U, F A}^{s}$, we simulate the protocol and send $\left(I D_{H A}, D I D_{M U}, A_{1}, T_{1}\right)$ to $\mathcal{A}$, where $A_{1}=h\left(I D_{M U}\left\|\alpha K_{F} P\right\| I D_{H A} \| T_{1}\right)$. We also imbed $\alpha P$ into the protocol and substitute $\alpha P$ in game $G_{4}$ for $\alpha P$. Now a random oracle is used to output a random value as the values $\alpha$ and $\beta$ are unknown. Therefore, we cannot compute $\alpha \beta P, \alpha K_{F} P$ as well as the session key. Suppose the output of the random oracle is $h$, then we let the session key $S K_{M F}=h\left(I D_{M U}\|\alpha \beta P\| \alpha K_{F} P\left\|h\left(X_{i} \| \gamma\right)\right\| T_{1} \| T_{4}\right)$. In such case, if $\mathcal{A}$ has computed the real session key himself, i.e., he has computed $S K_{M F}=h\left(I D_{M U}\|\alpha \beta P\| \alpha K_{F} P\left\|h\left(X_{i} \| \gamma\right)\right\| T_{1} \| T_{4}\right)$, we can obtain $\alpha \beta P$ and solve the ECDH problem by using $\mathcal{A}$ as a subroutine. Now we see, the games $G_{5}$ and $G_{4}$ are indistinguishable unless $\mathcal{A}$ has queried the random oracles $h$ on $S K_{M F}=h\left(I D_{M U}\|\alpha \beta P\| \alpha K_{F} P\left\|h\left(X_{i} \| \gamma\right)\right\| T_{1} \| T_{4}\right)$. We bound the probability of this event by $q_{h} A d v_{\mathcal{A}}^{E C D H}(t)$. Then we have:

$$
\begin{aligned}
& \operatorname{Diff}_{5}=\left|\operatorname{Pr}\left[\mathrm{Succ}_{5}\right]-\operatorname{Pr}\left[\mathrm{Succ}_{4}\right]\right| \\
& \leq q_{h} A d v_{\mathcal{A}}^{E C D H}\left(t+\left(1+q_{\text {exec }}+q_{\text {send }}\right) . \tau_{G}\right) .
\end{aligned}
$$

It is clear from the above game $G_{5}$, there is no collisions on the hash function and encryption / decryption query and no collision on the transcripts of the instances. Therefore, $\mathcal{A}$ does not guess the password correctly and is unable to solve the ECDH problem. Therefore, in random oracle model, all sessions are independent of each other. The adversary $\mathcal{A}$ cannot get any advantage in game $G_{5}$. Hence, we have: $\operatorname{Pr}\left[\operatorname{Succ}_{5}\right]=1 / 2$.

From definition 2,3 definition 4, consequently from the aforementioned equations, one can get the result 
of Theorem 1:

$q_{h} A d v_{\mathcal{A}}^{E C D H}\left(t+\left(1+q_{\text {exec }}+q_{\text {send }}\right) \cdot \tau_{G}\right)$

is negligible because $A d v_{\mathcal{A}}^{E C D H}(t)$ (the probability of breaking ECDH problem) is negligible;

$q$ is a large prime and $l$ is the security parameter. The time complexity of $q_{\text {send }}, q_{\text {exec }}$ and $q_{\text {hash }}$ are considered to be executed in polynomial time. Therefore,

$\frac{2 q_{\text {send }}}{\left|D_{P W}\right|}+\frac{\left(q_{E / D}^{2}+q_{\text {send }}+q_{\text {exec }}\right)^{2}}{2(q-1)}+\frac{q_{\text {hash }}^{2}+2\left(q_{\text {send }(B i o)}+q_{\text {send }}+q_{\text {exec }}\right)}{2^{l}}$ is negligible.

Hence the proof.

\section{Discussions on Some Attacks}

\subsection{Stolen Smartcard Attack}

In this case, either the smartcard of the user is stolen or found by an attacker. Then the information stored in the smartcard $\left\{I D_{H A}, Z_{i}, L_{i}, V_{i}\right\}$ can be extracted by the power analysis attack $[7-8,16,20]$ as described in the adversary model. In order to login and enjoy the services of $F A$, a valid user $I D_{M U}, H(B i o)$ and $P W_{M U}$ should be provided by the attacker. However, we show that the login credentials cannot be obtained by an attacker. The details are as follows:

\section{a Offline Password/Biometric Key Guessing Attack}

Usingtheoffline method, an attackerwantstoguess the user's password/biometric key. But due to the known fact that the user's biometrickey cannotbeguessed, $\mathcal{A}$ tries to guess user's password $P W_{M U}$ which was used in the computation of $m=L_{i} \oplus h\left(H(B i o) \| I D_{M U}\right)$, and $V=h\left(P W_{M U} \oplus m \oplus I D_{M U}\right)$. Therefore, to guess the password correctly, the attacker has to correctly guess the login credentials $I D_{M U}$ and $P W_{M U}$ concurrently, but the probability of guessing $I D_{M U}$ of length exactly $l$ bits and $P W_{M U}$ of length exactly $n$ characters correctly at the same time is approximately $\frac{1}{2^{6 n+l}}$ which is negligible. Thus, it is not possible to guess the user's password/biometric key in polynomial time. Hence, our proposed scheme withstands the offline password/ biometric key guessing attack.

\section{b User Anonymity}

The user's real identity $I D_{M U}$ is preserved by mask- ing it with the master secret key of GWN. Therefore, when a user enters the roaming region with different $F A$ networks, $M U$ provides the pseudo-identity $D I D_{M U}$ to $F A$. The user's identity is encrypted with the elliptic point $\alpha K_{F} P$, thereby it is evident that only the participants can decrypt and obtain the user's real identity.

\section{c User's Traceability}

When a user enters the roaming region with different $F A$ networks, $M U$ is allowed to provide his/her pseudo-identity which was transmitted during the login request $m_{1}$. The pseudo-identity varies each time the user tries to get access $F A$. This pseudo-identity works as a temporary identity of the MU and can be dynamically updated as and when the $M U$ visits different FAs. Thus, user traceability cannot be achieved.

\subsection{Privileged Insider Attack}

The password, $P W_{M U}$ of $M U$ is not sent as plaintext. $M U$ computes and sends $Y_{i}=h\left(P W_{M U} \| m\right)$ by using a random $m \in Z_{P}^{*}$. Therefore, an insider at $H A$ fails to obtain $M U$ s password from a registration request phase. In addition, due to the non-invertible nature of one-way hash function retrieving $P W_{M U}$ from $Y_{i}$ is computationally infeasible. Therefore, our proposed scheme resists the insider attack.

\subsection{Replay Attack}

As described in our proposed scheme, every transmitted message is included with a built-in timestamp of $M U$, and $F A$. The legal participants could figure out the replay attack by checking the freshness of the incoming message. Thus our proposed scheme can resist the replay attack.

\subsection{Impersonation Attack}

In the roaming authentication, we need to consider three scenarios of impersonation attack, i.e., on $M U$, on visiting $F A$, and finally on $H A$. The details are as follows:

$1 M U$ : Suppose, the adversary $\mathcal{A}$ wishes to enjoy the services from $F A$ on behalf of $M U$. Firstly, $\mathcal{A}$ must overcome the authentication process by $H A$. It is evident that $\mathcal{A}$ does not possess $M U$ 's secret $\alpha K_{F} P$. Therefore, a trial to forge $A_{1}$ fails to pass the authentication process by $F A$. Thus, this shows that $\mathcal{A}$ cannot impersonate $M U$. 
$2 F A / H A$ : It is observed that, in our proposal, each $\beta P$ value computed by $F A$ is sent to $H A$ which cannot be extracted by $\mathcal{A}$ as the communication between $F A$ and $H A$ is over a secure channel. Furthermore, the same $\beta P$ value is sent to $M U$ by masking the elliptic point and compute $D_{F A}$ and $A_{2}$ using the secret keys $K_{F H}$ and $\beta K_{H} P$. Thus, to impersonate $F A / H A, \mathcal{A}$ needs to know or extract the secret keys. As the key $K_{F H}$ is kept secret to $F A$ and $H A$, extracting the shared secret key by $\mathcal{A}$ is computationally infeasible. Moreover, $\mathcal{A}$ does not know $H A$ 's master key $K_{H}$, so $S_{M H}$ cannot be computed. Thus, $\mathcal{A}$ fails to impersonate $H A$ to $M U$ or $H A$ to $F A$.

\subsection{Perfect Forward Secrecy}

Suppose $\mathcal{A}$ wants to compute the session key $S K_{M F}=h\left(I D_{M U}\|\alpha \beta P\| \alpha K_{H} P\left\|h\left(X_{i} \| \gamma\right)\right\| T_{1} \| T_{4}\right)$ so as to communicate with the roaming network for timely communication. However, in order to compute the session key, $\mathcal{A}$ needs to compute $\alpha \beta P$ and $\alpha K_{F} P$ although $\alpha P$ and $\beta P$ are known which is an ECDH problem. Thus, the session key is not acquired. As a result, the forward secrecy is provided in the proposed scheme.

\subsection{Fair Key Agreement}

Our proposed scheme ends up with $M U$ and $F A$ agreeing on shared session key $S K_{M F}$ where both the parties ensure equal contribution. i.e., the session key $S K_{M F}=h\left(I D_{M U}\|\alpha \beta P\| \alpha K_{H} P\left\|h\left(X_{i} \| \gamma\right)\right\| T_{1} \| T_{4}\right)$ is computed using the random points with individual identities produced by $M U$ and $F A$, respectively. Such as, $\alpha \beta P, I D_{M U}$ and $\alpha K_{H} P$ are the random number points with identities produced by $M U$ and $F A$. This clearly signifies the equal contribution of the participants $(M U / F A)$. In this way, our key agreement protocol can assure the fairness property.

\section{6.\%. Mutual Entity Authentication}

Our proposed scheme supports mutual authentication among $M U, F A$ and $H A$.

$1 F A$ authenticates $M U$ by checking the validity of the timestamp $T_{1}$ and $I D_{M U}$ by applying verification on $A_{1} \stackrel{?}{=} h\left(I D_{M U}\left\|\alpha K_{F} P\right\| I D_{H A} \| T_{1}\right)$.

$2 F A$ computes $\beta P$ and $D_{F A}$ by encrypting with the shared key $K_{F H}$. Once the mes- sage $m_{3}=\left\{D_{H A}\right.$, Auth $\left._{H A}\right\}$ is received, FA decrypts $D_{H A}$ and validates $\beta P$ by verification on $A_{3} \stackrel{?}{=} h\left(h\left(X_{i} \| \gamma\right)\|\beta P\| K_{F H}\left\|D_{H A}\right\| T_{3}\right)$ is right. If this verification holds, $F A$ authenticates $H A$ directly and computes the session key $S K_{M F}$ and $A_{4}$.

$3 M U$ verifies the authenticity of $A_{4}=h\left(I D_{F A}\left\|S K_{M F}\right\| T_{4} \| \gamma\right)$ by computing $S K_{M F}$. If this verification holds, $M U$ authenticates $F A$ and $H A$, respectively.

Table 2

Comparison of security features

\begin{tabular}{c|c|c|c|c|c|c}
\hline $\begin{array}{c}\text { Security } \\
\text { attributes }\end{array}$ & Our & {$[34]$} & {$[26]$} & {$[30]$} & {$[13]$} & {$[22]$} \\
\hline$S_{1}$ & $\sqrt{ }$ & $\times$ & $\sqrt{ }$ & $\times$ & $\sqrt{ }$ & $\sqrt{ }$ \\
\hline$S_{2}$ & $\sqrt{ }$ & $\sqrt{ }$ & $\times$ & $\sqrt{ }$ & $\times$ & $\times$ \\
\hline$S_{3}$ & $\sqrt{ }$ & $\times$ & $\times$ & $\times$ & $\sqrt{ }$ & $\times$ \\
\hline$S_{4}$ & $\sqrt{ }$ & $\times$ & $\sqrt{ }$ & $\times$ & $\sqrt{ }$ & $\sqrt{ }$ \\
\hline$S_{5}$ & $\sqrt{ }$ & $\times$ & $\times$ & $\times$ & $\times$ & $\times$ \\
\hline$S_{6}$ & $\sqrt{ }$ & $\times$ & $\sqrt{ }$ & $\sqrt{ }$ & $\times$ & $\times$ \\
\hline$S_{7}$ & $\sqrt{ }$ & $\sqrt{ }$ & $\sqrt{ }$ & $\times$ & $\sqrt{ }$ & $\times$ \\
\hline$S_{8}$ & $\sqrt{ }$ & $\times$ & $\sqrt{ }$ & $\sqrt{ }$ & $\sqrt{ }$ & $\sqrt{ }$ \\
\hline$S_{9}$ & $\times$ & $\times$ & $\sqrt{ }$ & $\sqrt{ }$ & $\times$ & $\times$ \\
\hline$S_{10}$ & $\sqrt{ }$ & $\sqrt{ }$ & $\times$ & $\times$ & $\times$ & $\sqrt{ }$ \\
\hline$S_{11}$ & $\sqrt{ }$ & $\times$ & $\times$ & $\times$ & $\sqrt{ }$ & $\times$ \\
\hline$S_{12}$ & $\sqrt{ }$ & $\sqrt{ }$ & $\times$ & $\times$ & $\times$ & $\sqrt{ }$ \\
\hline
\end{tabular}

Note: $\sqrt{ }=$ preserved; $\times=$ not preserved.

$S_{1}$ : Resists password guessing attack; $S_{2}$ : Protects privileged-insider attack; $S_{3}$ : Provides user anonymity; $S_{4}$ : Resilient against stolen smartcard attack; $S_{5}$ : Secure against impersonation attack; $S_{6}$ : Protects replay attack; $S_{7}$ : Provides proper mutual authentication; $S_{8}$ : Provides two-factor security; $S_{9}$ : $H A$ knows the session key; $S_{10}$ : Provides perfect forward secrecy; $S_{11}$ : Restricts user traceability; $S_{12}$ : Discloses session key. 


\section{Performance Comparison with Related Schemes}

In this section, the performance and functionality features of the proposed scheme are compared with the existing similar authentication schemes proposed for the GLOMONET.

\subsection{Security Features Comparison}

In Table 3, the security features provided and protected by our proposed scheme are compared with the existing similar schemes [13, 22, 26, 30, 34]. As compared to existing similar schemes, our proposed scheme protects various known attacks and also supports various good features.

\subsection{Performance Analysis}

In Table 4, according to [24-25, 35], the time consumed by the cryptographic one-way hash function $T_{h}=0.0023 \mathrm{~ms}$ and the symmetric encryption/decryption operations $T_{\Omega}=0.0046 \mathrm{~ms}$ were considered. In Table 5, the computational time cost and communication overheads required during the login and authentication phases are compared with the existing similar schemes. The output of the one-way hash function $h(\cdot)$ is $128 \mathrm{bits}$, if SHA-1 hashing algorithm
[3] and symmetric encryption with 256 bits is used. Further, we assume that each timestamp, random nonce/random number, identity of ( $M U, F A, H A)$ 's is 160 bits in length.

The key points are as follows:

- From Fig. 6, we are able to show that our scheme takes more storage space in comparison to [13], but takes less storage space than [22, 26, 30, 34].

_ Although our scheme takes more computation cost as compared to [26] and nearly equivalent computation cost as compared to [22,34], but has better computation cost as compared to $[13,30]$.

_ From Fig. 6, we are able to illuminate that the communication cost of the proposed scheme is higher than [26], but less as compared to [13, 22, 30, 34].

_ The most important part is the security. The proposed scheme has better security features by overcoming the drawbacks as compared to existing similar schemes. We have also presented our scheme with a formal verification.

Therefore, Table 3, Table 5 and Fig. 6 show that the proposed scheme is better as compared to existing similar schemes in security, computation cost and communication cost.

Table 3

Computation cost analysis [24-25, 35]

\begin{tabular}{c|l|l}
\hline Notation & \multicolumn{1}{|c}{ Description } & $\approx$ execution time \\
\hline$T_{h}$ & One-way hash function & $0,0023 \mathrm{~ms}$ \\
\hline$T_{\Omega}$ & Symmetric key encryption/decryption & $0,0046 \mathrm{~ms}$ \\
\hline$T_{E M}$ & Elliptic curve point multiplication & $2,2260 \mathrm{~ms}$ \\
\hline$T_{M}$ & 1024 -bit modular exponentiation & $3,8500 \mathrm{~ms}$ \\
\hline
\end{tabular}

Table 4

Performance comparison among our scheme and other schemes

\begin{tabular}{|c|c|c|c|c|c|c|}
\hline Scheme & [13] & {$[30]$} & [26] & {$[22]$} & [34] & Our \\
\hline Storage in Smartcard(bytes) & 52 & 108 & 160 & 92 & 108 & 68 \\
\hline \multirow[t]{2}{*}{$\begin{array}{l}\text { Computation cost } \\
\text { and time for Login } \\
\text { and Authentication }\end{array}$} & $\begin{array}{l}3 T_{h}+T_{M} \\
4 T_{h} \\
5 T_{h}+2 T_{M}\end{array}$ & $\begin{array}{l}4 T_{h}+T_{M} \\
4 T_{h}+T_{M} \\
5 T_{h}+2 T_{M}\end{array}$ & $\begin{array}{l}8 T_{h} \\
1 T_{h}+2 T_{\Omega} \\
4 T_{h}+2 T_{\Omega}+1 T_{M}\end{array}$ & $\begin{array}{l}5 T_{h}+2 T_{E M} \\
4 T_{h}+2 T_{E M} \\
5 T_{h}\end{array}$ & $\begin{array}{l}1 T_{h}+2 T_{\Omega}+2 T_{E M} \\
2 T_{h}+2 T_{\Omega}+2 T_{E M} \\
1 T_{h}+6 T_{\Omega}\end{array}$ & $\begin{array}{l}5 T_{h}+2 T_{E M} \\
4 T_{h}+2 T_{\Omega}+2 T_{E M} \\
3 T_{h}+2 T_{\Omega}\end{array}$ \\
\hline & $\begin{array}{l}12 T_{h}+3 T_{M} \\
\approx 11.5776\end{array}$ & $\begin{array}{l}13 T_{h}+4 T_{M} \\
\approx 15.4299\end{array}$ & $\begin{array}{c}13 T_{h}+4 T_{\Omega}+1 T_{M} \\
\quad \approx 3.8983\end{array}$ & $\begin{array}{l}14 T_{h}+4 T_{E M} \\
\quad \approx 8.9362\end{array}$ & $\begin{array}{c}4 T_{h}+10 T_{\Omega}+4 T_{E M} \\
\approx 8.9592\end{array}$ & $\begin{array}{c}12 T_{h}+4 T_{\Omega}+4 T_{E M} \\
\approx 8.95\end{array}$ \\
\hline Communication cost(bytes) & 420 & 420 & 176 & 292 & 332 & 272 \\
\hline Formal verification & No & No & No & No & Yes & Yes \\
\hline Security & No & No & No & No & No & Yes \\
\hline
\end{tabular}




\section{Figure 4}

Simulation result for our proposed scheme

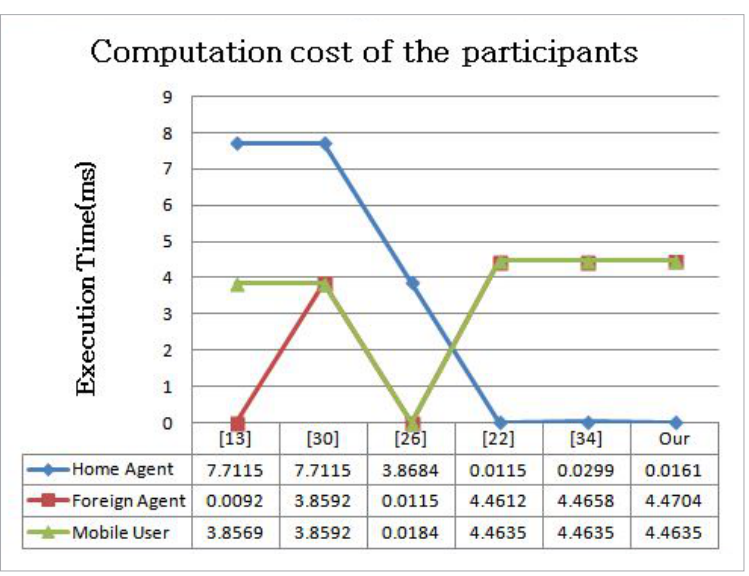

(a) The result of individual computation costs of the involved parties

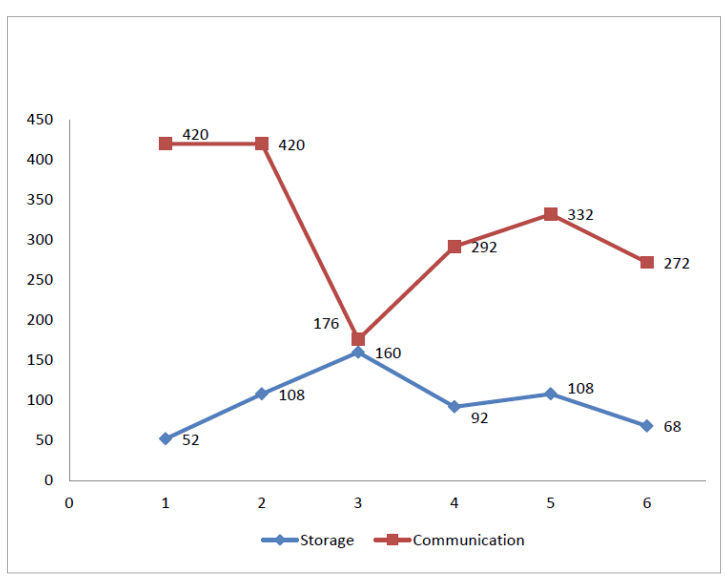

(b) The result of Storage and Communication costs among the proposed schemes

\section{Conclusion}

In this paper, we have reviewed the well designed Zhang et al.'s authentication scheme for GLOMONET, and presented various security pitfalls which include password guessing attack, impersonation attack, replay attack, user anonymity and traceability attack. To overcome the security weaknesses of Zhang et al.'s scheme, we have designed an authentication scheme for GLOMON-
ETs. The security analysis has been done using standard formal provable security proof. The informal cryptanalysis proofs to the resilience of relevant security attacks have been presented. The analysis of proposed scheme demonstrates that the proposed scheme addresses both security and privacy challenges. Results prove that the proposed scheme is efficient.

\section{References}

1. Alizadeh, M., Abolfazli, S., Zamani, M., Baharun, S., Sakurai, K. Authentication in Mobile Cloud Computing: A Survey. Journal of Network and Computer Applications, 2016, 61, 59-80. https://doi.org/10.1016/j. jnca.2015.10.005

2. Astorga, J., Aguado, M., Toledo, N., Higuero, M. A High Performance Link Layer Mobility Management Strategy for Professional Private Broadband Networks. Journal of Network and Computer Applications, 2013, 36(4), 1152-1163. https://doi.org/10.1016/j.jnca.2013.01.005

3. Burrows, J. H. Secure Hash Standard. FIPS PUB 180-1, National Institute of Standards and Technology (NIST), U.S. Department of Commerce, April 1995. http://csrc. nist.gov/publications/fips/fips180-2/fips180-2.pdf. Accessed on July 2015.
4. Chen, Y.-C., Chuang, S.-C., Yeh, L.-Y., Huang, J.-L. A Practical Authentication Protocol with Anonymity for Wireless Access Networks. Wireless Communications and Mobile Computing, 2011, 11(10), 1366-1375.https:// doi.org/10.1002/wcm.933

5. Chen, C., He, D., Chan, S., Bu, J., Gao, Y., Fan, R. Lightweight and Provably Secure User Authentication with Anonymity for the Global Mobility Network. International Journal of Communication Systems, 2011, 24(3), 347-362. https://doi.org/10.1002/dac.1158

6. Chang, C.-C., Tsai, H.-C. An Anonymous and Self-Verified Mobile Authentication with Authenticated Key Agreement for Large-Scale Wireless Networks. IEEE Transactions on Wireless Communications, 2010, 9(11), 33463353. https://doi.org/10.1109/TWC.2010.092410.090022 
7. Dolev, D., Yao, A. C. On the Security of Public Key Protocols. IEEE Transactions on Information Theory, 1983, 29(2), 198-208. https://doi.org/10.1109/TIT.1983.1056650

8. Eisenbarth, T., Kasper, T., Moradi, A., Paar, C. Salmasizadeh, M., Shalmani, M., Manzuri, T. On the Power of Power Analysis in the Real World: A Complete Break of the Keeloq Code Hopping Scheme. In Advances in Cryptology - CRYPTO-2008, Lecture Notes in Computer Science, Springer Berlin Heidelberg, 2008, 5157, 203-220.

9. Farash, M. S., Chaudhry, S. A., Heydari, M., Sadough, S., Mohammad, S., Kumari, S., Khan, M. K. A Lightweight Anonymous Authentication Scheme for Consumer Roaming in Ubiquitous Networks with Provable Security. International Journal of Communication Systems, 2015, 30(4), 1-20.

10. Gope, P., Hwang, T. An Efficient Mutual Authentication and Key Agreement Scheme Preserving Strong Anonymity of the Mobile User in Global Mobility Networks. Journal of Network and Computer Applications, 2016, 62,1-8. https://doi.org/10.1016/j.jnca.2015.12.003

11. He, D., Kumar, N., Khan, M. K., Lee, J.-H. Anonymous Two-Factor Authentication for Consumer Roaming Service in Global Mobility Networks. IEEE Transactions on Consumer Electronics, 2013, 59(4), 811-81\%. https://doi.org/10.1109/TCE.2013.6689693

12. He, D., Zhang, Y., Chen, J. Cryptanalysis and Improvement of an Anonymous Authentication Protocol for Wireless Access Networks. Wireless Personal Communications, 2014, 74(2), 229-243. https://doi. org/10.1007/s1127r7-013-1282-x

13. Jiang, Q., Ma, J., Li, G., Yang, L. An Enhanced Authentication Scheme with Privacy Preservation for Roaming Service in Global Mobility Networks. Wireless Personal Communications, 2013, 68(4), 1477-1491. https://doi. org/10.1007/s1127r7-012-0535-4

14. Jin, A. T. B., Ling, D. N. C., Goh, A. Biohashing: Two Factor Authentication Featuring Fingerprint Data and Tokenised Random Number. Pattern Recognition, 2004, 37(11), 2245-2255. https://doi.org/10.1016/j.patcog.2004.04.011

15. Kim, J.-S., Kwak, J. Improved Secure Anonymous Authentication Scheme for Roaming Service in Global Mobility Networks. International Journal of Security and Its Applications, 2012, 6(3), 45-54.

16. Kocher, P., Jaffe, J., Jun, B. Differential Power Analysis. In Proceedings of Advances in Cryptology - CRYPTO’99, Lecture Notes in Computer Science, Springer Berlin Heidelberg, 1999, 1666, 388-397. https://doi. org/10.1007/3-540-48405-1_25
17. Kumar, V., Jangirala, S., Ahmad, M. An Efficient Mutual Authentication Framework for Healthcare System in Cloud Computing. Journal of Medical Systems, 2018, 42(8), 1-25. https://doi.org/10.1007/s10916-018-0987-5

18. Lee, C.-Y., Chang, C.-C., Lin, C.-H. User Authentication with Anonymit for Global Moblity Networks. In 2nd International Conference on Mobile Technology, Applications and Systems, IEEE, 2005, 1-5.

19. Lee, C.-C., Hwang, M.-S., Liao, I.-E. Security Enhancement an a New Authentication Scheme with Anonymity for Wireless Environments. IEEE Transactions on Industrial Electronics, 2006, 53(5), 1683-1687. https:// doi.org/10.1109/TIE.2006.881998

20. Messerges, T. S., Dabbish, E. A., Sloan, R. H. Examining Smart-Card Security Under the Threat of Power Analysis Attacks. IEEE Transactions on Computers, 2002, 51(5), 541-552. https://doi.org/10.1109/ TC.2002.1004593

21. Modares, H., Moravejosharieh, A., Lloret, J., Salleh, R. A Survey of Secure Protocols in Mobile IPv6. Journal of Network and Computer Applications, 2014, 39, 351368. https://doi.org/10.1016/j.jnca.2013.07.013

22. Mun, H., Han, K., Lee, Y. S., Yeun, C. Y., Choi, H. H. Enhanced Secure Anonymous Authentication Scheme for Roaming Service in Global Mobility Networks. Mathematical and Computer Modelling, 2012, 55(1), 214-222. https://doi.org/10.1016/j.mcm.2011.04.036

23. Nanni, L., Brahnam, S., Lumini, A. Biohashing Applied to Orientation-Based Minutia Descriptor for Secure Fingerprint Authentication System. Electronics Letters, 2011, 47(15), 851-853. https://doi.org/10.1049/ el.2011.1525

24. Niinuma, K., Park, U., Jain, A. K. Soft Biometric Traits for Continuous User Authentication. IEEE Transactions on Information Forensics and Security, 2010, 5(4), 771-780. https://doi.org/10.1109/TIFS.2010.2075927

25. Odelu, V., Das, A. K., Goswami, A. An Efficient Biometric-Based Privacy-Preserving Three-Party Authentication with Key Agreement Protocol Using Smart Cards. Security and Communication Networks, 2015, 8(18), 4136-4156. https://doi.org/10.1002/sec.1330

26. Shin, S., Yeh, H., Kim, K. An Efficient Secure Authentication Scheme with User Anonymity for Roaming User in Ubiquitous Networks. Peer-to-peer Networking and Applications, 2015, 8(4), 674-683. https://doi. org/10.1007/s12083-013-0218-2

27. Srinivas, J., Mishra, D., Mukhopadhyay, S. A Mutual Authentication Framework for Wireless Medical Sen- 
sor Networks. Journal of Medical Systems, 2017, 41(5), 1-19. https://doi.org/10.1007/s10916-017-0720-9

28. Srinivas, J., Mukhopadhyay, S., Mishra, D. Secure andEfficient User Authentication Scheme for Multi-Gateway Wireless Sensor Networks. Ad Hoc Networks, 2017, 54, 147-169. https://doi.org/10.1016/j.adhoc.2016.11.002

29. Suzuki, S., Nakada, K. An Authentication Technique Based on Distributed Security Management for the Global Mobility Network. IEEE Journal on Selected Areas in Communications, 1997, 15(8), 1608-1617. https:// doi.org/10.1109/49.634798

30. Wen, F., Susilo, W., Yang, G. A Secure and Effective Anonymous User Authentication Scheme for Roaming Service in Global Mobility Networks. Wireless Personal Communications, 2013, 73(3), 993-1004. https://doi. org/10.1007/s11277-013-1243-4

31. Wu, C.-C., Lee, W.-B., Tsaur, W.-J. A Secure Authentication Scheme with Anonymity for Wireless Communications. IEEE Communications Letters, 2008,12(10), 722723. https://doi.org/10.1109/LCOMM.2008.080283

32. Wu, F., Xu, L., Kumari, S., Li, X. A Privacy-Preserving and Provable User Authentication Scheme for Wireless
Sensor Networks based on Internet of Things Security. Journal of Ambient Intelligence and Humanized Computing, 2016, 1-16.

33. Xie, Q., Hu, B., Tan, X., Bao, M., Yu, X. Robust Anonymous Two-Factor Authentication Scheme for Roaming Service in Global Mobility Network. Wireless Personal Communications, 2014, 74(2), 601-614. https://doi. org/10.1007/s11277-013-1309-3

34. Zhang, G., Fan, D., Zhang, Y., Li, X., Liu, X. A Privacy Preserving Authentication Scheme for Roaming Services in Global Mobility Networks. Security and Communication Networks, 2015, 8(16), 2850-2859. https://doi. org/10.1002/sec.1209

35. Zhang, Q., Yin, Y., Zhan, D.-C., Peng, J. A Novel Serial Multimodal Biometrics Framework Based on Semisupervised Learning Techniques. IEEE Transactions on Information Forensics and Security, 2014, 9(10), 16811694. https://doi.org/10.1109/TIFS.2014.2346703

36. Zhu, J., Ma, J. A New Authentication Scheme with Anonymity for Wireless Environments. IEEE Transactions on Consumer Electronics, 2004, 50(1), 231-235. https:// doi.org/10.1109/TCE.2004.1277867 